\title{
On Effect of Equilibrium Fluctuations on Superfluid Density in Layered Superconductors
}

\author{
S.N. Artemenko and S.V.Remizov \\ Institute for Radioengineering and Electronics of Russian Academy of Sciences, Mokhovaya 11, 103907 Moscow, Russia
}

(October 28, 2018)

\begin{abstract}
We calculate suppression of inter- and intralayer superconducting currents due to equilibrium phase fluctuations and find that, in contrast to a recent prediction, the effect of thermal fluctuations cannot account for linear temperature dependence of the superfluid density in high- $\mathrm{T}_{c}$ superconductors at low temperatures. Quantum fluctuations are found to dominate over thermal fluctuations at low temperatures due to hardening of their spectrum caused by the Josephson plasma resonance. Near $\mathrm{T}_{c}$ sizeable thermal fluctuations are found to suppress the critical current in the stack direction stronger, than in the direction along the layers. Fluctuations of quasiparticle branch imbalance make the spectral density of voltage fluctuations at small frequencies non zero, in contrast to what may be expected from a naive interpretation of Nyquist formula.
\end{abstract}

PACS numbers: 74.40.+k, 74.25.-q, 74.25.Fy

One of important problems in layered high- $\mathrm{T}_{c}$ cuprate superconductors that is still under discussion is the origin of the observed linear low-temperature dependence of the superfluid density (phase stiffness). The latter is directly related to magnetic penetration depths, $\lambda_{\|}$and $\lambda_{\perp}$, for a magnetic field screened by currents flowing in directions parallel and perpendicular to the superconducting planes, respectively [1]. This dependence is usually attributed to contribution of quasiparticles near the nodes of the $d$-wave gap. According to the alternative explanation suggested in refs. [2,3] the linear decrease in the temperature dependence of $1 / \lambda^{2}$ is induced entirely by classical thermal phase fluctuations. Recently the role of fluctuations was reconsidered [4] for a $d$-wave superconductor by means of a microscopic approach within a functional integral framework, and quantum phase fluctuations were found to lead to a sizeable renormalization of the superfluid density, the effect of thermal fluctuations being small for $T<T_{c}$.

Thermal and quantum fluctuations considered in refs. 2, 24 are equilibrium fluctuations, therefore, the problem can be solved by means of a phenomenological approach based on the fluctuation-dissipation theorem. Such an approach is more simple and physically transparent, being in the same time more general because it is not restricted to a specific model of high- $\mathrm{T}_{c}$ superconductivity. In the present work we reconsider the role of equilibrium phase fluctuations in layered superconductors applying the fluctuation-dissipation theorem to equations of the linear response, and come to conclusions different from those of refs. [2 4 ]. Our calculations demonstrate that, in agreement with the results of ref. [4], at low temperatures quantum fluctuations dominate over thermal fluctuations due to Coulomb effects. This happens because non-uniform phase fluctuations induce a fluctuating electric field resulting in a finite energy of such fluctuations which is reflected in a finite value of the Josephson plasma frequency. So unlike refs. [2,3] we assert that a contribution of thermal fluctuations cannot account for temperature dependence of the penetration depth. However, in contrast to the results of ref. [4] we find that renormalization of the superfluid density due to fluctuations at low temperatures is not large. Near $\mathrm{T}_{c}$, when quasiparticle density dominates over the superfluid density, we find a different picture. In this case thermally induced fluctuations of the quasiparticle branch imbalance are found to be important, and a reduction of the superfluid density may become sizeable. We find that a suppression of the superconducting critical current due to thermal fluctuations is larger in the stack direction, than in the direction parallel to the conducting layers. This points out a possibility of a destruction of the superconductivity in the perpendicular direction at temperatures lower, than the critical temperature for the in-plane direction.

The fluctuation-dissipation theorem relates correlation functions of fluctuations to dissipative parts of kinetic coefficients describing the linear response of a system. So we start with the linear response of a layered superconductor. We consider expressions for current and charge density written in a form of functions of gauge invariant vector and scalar potentials. These potentials are

$$
\mathbf{P}_{s}=(1 / 2) \nabla \chi-(1 / c) \mathbf{A}, \quad \mu=(1 / 2) \partial_{t} \chi+\Phi,
$$

where $\mathbf{A}$ is the vector potential, $\chi$ is the phase of the order parameter, and $\Phi$ is the electric potential (we use units with $e=1, \hbar=1$, and $k_{B}=1$ ). The gauge invariant vector potential plays a role of the superconducting momentum, while the gauge invariant scalar potential is responsible for branch imbalance [5] and charging effects. The electric field is expressed in terms of the potentials as

$$
\mathbf{E}=\partial_{t} \mathbf{P}_{s}-\nabla \mu .
$$


In layered superconductors the variables above are related to specific layers and must be supplied by layer numbers, e. g., the parallel component of $\mathbf{P}_{s}$ will be denoted as $\mathbf{P}_{n}$. Furthermore, the role of the perpendicular component of $\mathbf{P}_{s}$ plays the gauge invariant phase difference between the layers, $\varphi_{n}=\chi_{n+1}-\chi_{n}-2 s A_{\perp} / c$, where $s$ is the lattice period in the stack direction. So, we may denote $P_{\perp n}=\varphi_{n} / 2 s$.

Equations determining current and charge densities read

$$
\begin{aligned}
& \mathbf{j}_{\| n}=\frac{c^{2}}{4 \pi \lambda_{\|}^{2}} \mathbf{P}_{n}+\sigma_{0 \|} \partial_{t} \mathbf{P}_{n}-\sigma_{1 \|} \nabla_{\|} \mu_{n} \\
& j_{\perp n}=j_{c} \sin \varphi_{n}+\frac{\sigma_{0 \perp}}{2 s} \partial_{t} \varphi_{n}-\sigma_{1 \perp} \nabla_{n} \mu_{n}, \\
& \partial_{t} \rho_{n}=\left(\gamma \partial_{t}+\nu_{b}\right) \frac{\kappa^{2}}{4 \pi} \mu_{n}-\left(\sigma_{2 \|} \nabla_{\|}^{2}+\sigma_{2 \perp} \nabla_{n}^{2}\right) \mu_{n}+ \\
& \partial_{t}\left(\sigma_{1 \|} \nabla_{\|} \mathbf{P}_{n}+\sigma_{1 \perp} \nabla_{n} \varphi_{n} / 2 s\right),
\end{aligned}
$$

where $\nabla_{n} \mu_{n}=\left(\mu_{n+1}-\mu_{n}\right) / s$ is the discrete version of the spatial derivative in the transverse direction, $\kappa^{-1}$ is the Thomas-Fermi screening radius, and $\nu_{b}$ is the branch imbalance relaxation rate.

The first terms in equations (3⿻) describe the superconducting current for relative directions, and the rest terms are related for quasiparticle contributions. Equation (5) for charge density can be interpreted as a continuity equation for quasiparticles. It was derived for conventional superconductors in ref. [6] and generalized later for layered superconductors with $s$-wave [7] and $d$ wave [8] pairing.

Note that the quasiparticle current does not have the form $j=\sigma E$ valid for a normal state. Instead, the response of a superconductor to the components of the field $E$ expressed in terms of the temporal derivative of $\mathbf{P}_{s}$ and of the spatial derivative of $\mu$ ( $c f$. (2)) are described by different generalized conductivities $\sigma_{i}, i=0,1,2$. The respective parts of the electric field $E$ are induces by time dependent perturbations of current density, and by perturbations of charge density.

Explicit expressions for the coefficients in equations (35) depend on temperature, on scattering, and on a mechanism of the superconductivity (see [7,8], note that the explicit expressions for coefficients in ref. [8] were presented in the dynamic limit $\omega>D q^{2}$ where $D$ is a diffusion coefficient in quasiclassic approximation). At temperatures $T \rightarrow 0$ all conductivities are small $(e . g$. in a $s$-wave superconductor they are exponentially small), and $\gamma \rightarrow 1$. At $T \rightarrow T_{c}$ the conductivities $\sigma_{i}$ approach the normal state conductivities. Furthermore, the differences between $\sigma_{i}$ for a given direction vanishes as $\Delta / T$, $\gamma \rightarrow \Delta / T, \nu_{b} \rightarrow \Delta / T$, so that equation (5) approaches the continuity equation as $\Delta \rightarrow 0$. Strictly speaking products in (3) assume the convolution with respect to time and coordinates, $i$. e. in the Fourier transformed form the coefficients in the equations are frequency and wave vector dependent.

In order to derive equations relating fluctuations $\delta \mu$, $\delta \varphi$ and $\delta \mathbf{P}$ with fluctuations of charge and current densities, $\delta \rho$ and $\delta \mathbf{j}$, we insert expression (3-5) into Maxwell equation

$$
\nabla \times \mathbf{H}=\frac{4 \pi}{c} \mathbf{j}+\frac{1}{c} \partial_{t} \mathbf{D}
$$

and into the Poisson equation. Then making Fourier transformation with respect to time, to in-layer coordinates $\mathbf{r}_{\|}$, and to layer numbers $n$, we find

$$
\begin{aligned}
& \left(\begin{array}{c}
\delta j_{\perp} \\
\delta j_{\|} \\
\delta \rho
\end{array}\right)=\hat{A}\left(\begin{array}{c}
\delta \varphi / 2 s \\
\delta \mathbf{P}_{\|} \\
\delta \mu
\end{array}\right), \\
& \hat{A}=\frac{4}{\pi}\left(\begin{array}{ccc}
\epsilon\left(\omega \omega_{0}-\tilde{\omega}_{p}^{2}\right) & c^{2} q_{\|} \hat{q}_{\perp} & \epsilon \hat{q}_{\perp} \omega_{1} \\
c^{2} q_{\|} \hat{q}_{\perp} & \omega \Omega_{0}-\tilde{\Omega}_{p}^{2} & q_{\|} \Omega_{1} \\
\epsilon \hat{q}_{\perp} \omega_{1} & q_{\|} \Omega_{1} & \left(\gamma-\frac{\nu_{b}}{i \omega}\right) \kappa^{2}+\frac{\epsilon \hat{q}_{\perp}^{2} \omega_{2}+q_{\|}^{2} \Omega_{2}}{\omega}
\end{array}\right) .
\end{aligned}
$$

Here $\hat{q}_{\perp}=(2 / s) \sin \left(q_{\perp} s / 2\right)$, where $\left|q_{\perp}\right|<\pi / s$ is the wave number obtained from the discrete Fourier transformation with respect to layer numbers, $\omega_{i}=\omega+i \omega_{i r}, \Omega_{i}=$ $\omega+i \Omega_{i r}, \omega_{i r}=4 \pi \sigma_{i \perp} \epsilon$ and $\Omega_{i r}=4 \pi \sigma_{i \|}$ are dielectric relaxation frequencies. Furthermore, $\tilde{\omega}_{p}^{2}=\omega_{p}^{2}\left(1+\lambda_{\perp}^{2} q_{\|}^{2}\right)$, $\tilde{\Omega}_{p}^{2}=\Omega_{p}^{2}\left(1+\lambda_{\|}^{2} \hat{q}_{\perp}^{2}\right)$, where $\Omega_{p}=c / \lambda$ and $\omega_{p}=c / \lambda_{\perp} \sqrt{\epsilon}$ are the plasma frequencies for directions parallel and perpendicular to the layers, $\epsilon$ is a dielectric constant in transverse direction, $\lambda_{\perp}=c / \sqrt{8 \pi s j_{c}}$. The in-layer plasma frequency $\Omega_{p}$, is much larger than typical frequencies of the problem which are of order of the Josephson plasma frequency, $\omega_{p}$.

Note that matrix $\hat{A}$ satisfy the Onsager symmetry relations. Now we apply the fluctuation-dissipation theorem (cf. [9]) to equations (6).

From the expression for energy dissipation density $Q$ which in superconductors is given by 10 .

$$
Q=\rho \partial_{t} \mu+\mathbf{j} \partial_{t} \mathbf{P}_{s}
$$

one can identify potentials $\mu$ and $\mathbf{P}_{s}$ with generalized forces related to variables $\rho$ and $\mathbf{j}$, respectively. So according to the fluctuation-dissipation theorem correlation functions of $\delta j_{\perp}, \delta j_{\|}$and $\delta \rho$ are determined by imaginary parts of related coefficients of the matrix $\hat{A}$ in (6). For example, we find

$$
\left\langle\delta j_{\perp}(\mathbf{q}, \omega) \delta j_{\perp}\left(\mathbf{q}^{\prime}, \omega^{\prime}\right)\right\rangle=(2 \pi)^{4}\left(j_{\perp}^{2}\right)_{\omega} \delta\left(\omega+\omega^{\prime}\right) \delta\left(\mathbf{q}+\mathbf{q}^{\prime}\right)
$$

with $\left(j_{\perp}^{2}\right)_{\omega}=2 \tilde{T} \sigma_{0 \perp}^{\prime} / s$, where $\tilde{T}=(\omega / 2) \operatorname{coth}(\omega / 2 T)$, $\sigma^{\prime}=\Re \sigma$. Similarly, $\left(j_{\perp} \rho\right)_{\omega}=2 \tilde{T} \hat{q}_{\perp} \sigma_{1 \perp}^{\prime} / s \omega,\left(\rho^{2}\right)_{\omega}=$ $2 \tilde{T}\left(\nu_{b} / 4 \pi+q_{\perp}^{2} \sigma_{2 \perp}^{\prime}+q_{\|}^{2} \sigma_{2 \|}^{\prime}\right) / s \omega^{2}$ and so on. An alternative way to calculate correlation functions based on the Langevin sources in equations of motion, and on the dissipation function of the system gives similar results. 
Correlation functions of $\delta \mathbf{P}_{s}$ and $\delta \mu$ are related to the inverse matrix $\hat{A}^{-1}$. For example, using the upper diagonal component of $\hat{A}^{-1}$ we can find the spectral density of phase difference fluctuations

$$
\left(\delta \varphi^{2}\right)_{\omega}=\Im \frac{8 \pi \tilde{T}\left(\omega \Omega_{0}-\tilde{\Omega}_{p}^{2}\right)\left[\left(\gamma-\frac{\nu_{b}}{i \omega}\right) \kappa^{2}+\frac{\epsilon \hat{q}_{\perp}^{2} \omega_{2}+q_{\|}^{2} \Omega_{2}}{\omega}\right]}{D}
$$

where $D$ is the determinant of the matrix $\hat{A}$.

Zeros of $D$ determine collective modes and penetration of electric and magnetic fields into a superconductor. They are important in calculation of spectral density of voltage noise. The voltage between contacts separated by $N$ layers, can be expressed according to eq.(2) as $V=\partial_{t} \sum_{n=0}^{N-1} \varphi_{n} / 2-\left(\mu_{N}-\mu_{0}\right)$. Then we make Fourier transformation and calculate mean square value of the voltage using (9) and similar expressions for other correlation functions. We assume that the width of the contacts is large enough, therefore, we need functions at $q_{\|}=0$. Finally, after some algebra we find the expression for the spectral density of voltage fluctuations

$$
\begin{aligned}
& \left(\delta V^{2}\right)_{\omega}=\frac{16 \tilde{T}}{S \varepsilon} \int d q_{\perp} \frac{\sin ^{2} \frac{q_{\perp} L}{2}}{\hat{q}_{\perp}^{2}} \times \\
& \Im \frac{\omega\left(\omega \gamma+i \nu_{b}\right) \kappa^{2}+\epsilon \hat{q}_{\perp}^{2}\left[\omega\left(\omega_{0}+\omega_{2}-2 \omega_{1}\right)-\omega_{p}^{2}\right]}{\left(\omega_{p}^{2}-\omega \omega_{0}\right)\left(\omega \gamma+i \nu_{b}\right) \kappa^{2}+\epsilon \hat{q}_{\perp}^{2}\left[\omega\left(\omega_{1}^{2}-\omega_{0} \omega_{2}\right)+\omega_{2} \omega_{p}^{2}\right]}
\end{aligned}
$$

with $L=N s$.

At low temperatures, $T \ll \Delta$, the relaxation frequencies are small in comparison to plasma frequencies $\omega_{p}$, and zero of the denominator gives the underdamped Josephson plasma mode [7,11, 12 at $q_{\|}=0$. In the opposite limit $T \gg \Delta$, near $\mathrm{T}_{c}$, the plasma frequencies are small, $\Omega_{p}^{2}, \omega_{p}^{2} \propto N_{s} \propto(\Delta / T)^{2}$ where $N_{s}$ is a fraction of the condensed electrons. On the other hand, all conductivities $\sigma_{i \|}$ and $\sigma_{i \perp}$ at $T \rightarrow T_{c}$ approach the normal state conductivities for parallel and perpendicular directions, respectively. So near $\mathrm{T}_{c}$ the dielectric relaxation frequencies are larger, than plasma frequencies for respective directions. Furthermore, calculations for $s$ - and $d$ wave pairing give $\gamma=\pi \Delta / 4 T$ [6] and $\gamma=\pi \Delta_{0} / 2 T \sqrt{i \nu / \omega}$ [8], respectively. The branch imbalance relaxation rate is determined by energy relaxation for $s$-wave pairing and by elastic scattering for $d$-wave pairing, and in both cases $\nu_{b} \propto \Delta / T$. Then zeros of the denominator in equation (10) give the Carlson-Goldman mode 13] for direction perpendicular to the layers, that is underdamped in the case of isotropic pairing in a narrow frequency region, $\nu_{b}, \omega_{p}^{2} / \omega_{r} \ll \omega \ll(T / \Delta) \omega_{p}^{2} / \omega_{r}$. The spectrum of the mode is $\omega^{2} \approx \epsilon q_{\perp}^{2} \gamma / \kappa^{2}$. This spectrum in isotropic superconductors was found in clean limit in ref. [14] and in dirty limit in ref. [15]. Evolution of the spectrum from the Josephson plasma mode to the anisotropic CarlsonGoldman mode in layered superconductors was studied in ref. [7]. In the case of $d$-wave pairing $\gamma$ is not real, and the Carlson-Goldman mode is never underdamped ( $c f$. [8]).

For $N \gg 1$ the leading contribution to the voltage fluctuations is

$$
\left(\delta V^{2}\right)_{\omega}^{(0)}=\frac{2 \tilde{T} L}{S \sigma_{0 \perp}} \frac{\omega^{2} \omega_{0 r}^{2}}{\left(\omega_{p}^{2}-\omega^{2}\right)^{2}+\omega^{2} \omega_{0 r}^{2}} .
$$

This expression corresponds to the Nyquist formula and exhibits the Josephson plasma resonance. At zero frequency this contribution vanishes, but there is an additional contribution to voltage fluctuations which remains finite at low frequencies $\omega \ll \nu_{b}$. The latter contribution is related to the quasiparticle branch imbalance fluctuations, and is especially pronounced near $T_{c}$ :

$$
\left(\delta V^{2}\right)_{\omega}^{(1)}=\frac{4 T}{S \sigma_{\perp}} \frac{l_{E}^{2}}{\sqrt{4 l_{E}^{2}+s^{2}}},
$$

where $l_{E}^{2}=4 \pi \sigma_{\perp} / \nu_{b} \kappa^{2}$ (we assumed a thick sample, $L \gg l_{E}$ ). Equation (12) contradicts to a naive interpretation of the Nyquist theorem, according to which voltage noise at zero frequency is absent because the static resistivity of a superconductor is equal to zero. The non-zero contribution is related to a voltage drop near the superconductor boundary due to penetration of the electric field into layered superconductor over a distance needed for the branch imbalance relaxation. Though $\left(\delta V^{2}\right)_{\omega}$ in (12) is independent on the distance $L$ between the contacts, it may give a sizeable contribution which can be measured easily in small mesa structures.

Using equation (9) we can calculate mean square fluctuation of the phase difference.

$$
\left\langle\delta \varphi^{2}\right\rangle=\int\left(\delta \varphi^{2}\right)_{\omega} \frac{d \omega d \mathbf{q}}{(2 \pi)^{4}} .
$$

Since $\left\langle j_{c} \sin (\varphi+\delta \varphi)\right\rangle \approx j_{c}\left(1-\left\langle\delta \varphi^{2}\right\rangle / 2\right) \sin \varphi$, the latter term determines renormalization of the superfluid density. We calculate this renormalization, first, in the limit of low temperature.

At $T \ll \Delta$ the relaxation frequencies are small in comparison to the related plasma frequencies $\omega_{p}$ and $\Omega_{p}$. We assume a simplifying condition $\kappa s / \epsilon \gg 1$ which holds in high- $\mathrm{T}_{c}$ superconductors, therefore, perturbations of $\delta \mu$ are small and can be neglected. Then the leading terms in the denominator of the matrix $\hat{A}$ are

$$
\begin{gathered}
D=\omega \kappa^{2} \Omega_{p}^{2}\left\{\epsilon\left(\omega_{p}^{2}-\omega^{2}\right)\left(1+\lambda_{\|}^{2} \hat{q}_{\perp}^{2}\right)+c^{2} q_{\|}^{2}\right. \\
\left.-4 \pi i \omega\left[\sigma_{0 \perp}\left(1+\lambda_{\|}^{2} \hat{q}_{\perp}^{2}\right)+\sigma_{0 \|} \epsilon\left(\omega_{p}^{2}-\omega^{2}+c^{2} q_{\|}^{2} \epsilon\right) / \Omega_{p}^{2}\right]\right\} .
\end{gathered}
$$

A phase volume near zeros of $D$ related to the Josephson plasma mode contributes much to the integral (13) resulting in a finite fluctuations at $T=0$. Furthermore, the integral over $q_{\|}$diverges at large $q_{\|}$, and we cut-off it 
at $q_{\|} \sim 1 / \xi_{0}$, where $\xi_{0}$ is the superconducting correlation length. In dimensional units we obtain

$$
\begin{aligned}
& \left\langle\delta \varphi^{2}\right\rangle \approx \frac{16}{\pi \sqrt{\epsilon}} \frac{e^{2}}{\hbar c} \frac{\lambda_{\|}}{\xi_{0}}\left(1+\frac{T^{2}}{T_{0}^{2}}\right), \\
& T_{0} \approx \frac{\hbar \Omega_{p}}{2 \pi k_{B}} \sqrt{\frac{3 \Omega_{p} s}{2 \sigma_{0 \|} \xi_{0} \sqrt{\epsilon} \ln \lambda_{J} / \xi_{0}}} .
\end{aligned}
$$

This result differs from that obtained in refs. [2 1]. For parameter values typical for layered cuprates we find $T_{0} \gg T_{c}$. The magnitude of $\left\langle\delta \varphi^{2}\right\rangle$ is not large because the large factor $\lambda / \xi_{0}$ in (14) is multiplied by the small fine structure constant. Thus the renormalization of the penetration depths due to phase fluctuations at low temperatures is practically temperature independent, and is not large.

Calculation of $\left\langle\delta P_{\|}\right\rangle^{2} \xi_{0}^{2}$ which determines suppression of the superfluid density in the in-plane direction gives value similar to (14).

Now we calculate fluctuations at high temperatures, $T \rightarrow T_{c}$. Since at such temperatures fluctuations are quasi-stationary they can be found using the standard approach based on functional integration of the free energy of the system, which includes energy of the magnetic field and of the superconducting current. We calculate $\left\langle\delta \varphi^{2}\right\rangle$ and $\left\langle\delta P_{\|}^{2}\right\rangle \xi_{0}^{2}$, which determine the renormalization of the superfluid stiffness in perpendicular and parallel directions, respectively, using again the cut-off at $q_{\|} \sim 1 / \xi_{0}$. Then in the limit $\xi_{0} \ll \lambda_{J}$, which definitely holds for layered high- $\mathrm{T}_{c}$ superconductors, we find in dimensional units

$$
\left\langle\delta \varphi^{2}\right\rangle \approx \frac{16 e^{2} k_{B} T \lambda_{\|}^{2}}{\hbar^{2} c^{2} s} \ln \frac{\lambda_{\perp}}{\xi_{0}} \approx 4 \ln \frac{\lambda_{\perp}}{\xi_{0}}\left\langle\delta P_{\|}^{2}\right\rangle \xi_{0}^{2} .
$$

Since in layered high- $T_{c}$ superconductors $\lambda_{\perp}$ is by few orders of magnitude larger, than the correlation length $\xi_{0}$, the logarithm in equation (15) is large as well. Then suppression of the critical current by fluctuations in the direction perpendicular to the layers is larger, than in the parallel direction. Using parameters typical for BSCCO, $\lambda_{\|}(0) \approx 1.5 \times 10^{-5} \mathrm{~cm}, s \approx 1.5 \times 10^{-7} \mathrm{~cm}$ and $\lambda_{\perp} / \xi_{0} \approx$ $10^{4}$, we estimate

$$
\left\langle\delta \varphi^{2}\right\rangle \approx 0.5\left[\frac{\lambda_{\|}(T)}{\lambda_{\|}(0)}\right]^{2} \frac{T}{80 \mathrm{~K}} .
$$

Since $\left[\lambda_{\|}(T) / \lambda_{\|}(0)\right]^{2}$ diverges as $T \rightarrow T_{c}$ we conclude that thermal fluctuations may lead to a sizeable reduction of the critical current. Note that we study fluctuations in the linear approximation and, hence, do not take into account that the phase perturbations due to thermal fluctuations may overcome a finite potential barrier. The latter process would result in a destruction of a superconducting current analogous to Josephson junctions [16.
Observation of smaller values of $\mathrm{T}_{c}$ in the stack direction, than in direction parallel to the layers, was reported in YBCO single crystals with low oxygen content [17]. Such crystals are expected to have large $\lambda_{\|}$, which according to (15) is in favor of large fluctuations. However, it is difficult to explain by the fluctuation mechanism so large differences between critical temperatures observed in ref. 17] for different directions.

We are grateful to A. A. Varlamov for attracting our attention to the results of ref. [3] and for useful discussion, and to V. F. Gantmakher, K. E. Nagaev and A. Ya. Shulman for useful discussion. This work was supported by project 98-02-17221 of Russian Foundation for Basic Research, and by project 96053 of Russian program on superconductivity.

[1] W. N. Hardy et al., Phys. Rev. Lett. 70, 399 (1993); C. Panagopoulos, J. R. Cooper and T. Xiang, Phys. Rev. B 57, 13422 (1998).

[2] E. Roddik and D. Stoud, Phys. Rev. Lett. 74, 1430 (1995).

[3] E. W. Carlson, S. A. Kivelson, V. J. Emery and E. Manousakis, Phys. Rev. Lett. 83, 612 (1999).

[4] Arun Paramekanti, Mohit Randeria, T.V. Ramakrishnan and S.S. Mandal, cond-mat/0002349.

[5] M. Tinkham, Introduction to Superconductivity (McGrow-Hill, New York, 1996), and references therein.

[6] S. N. Artemenko, A. F. Volkov, Uspekhi Fiz. Nauk 128, 3 (1979) [Sov. Phys. Usp. 22, 295 (1979)].

[7] S. N. Artemenko and A. G. Kobel'kov, Pis'ma Zh. Eksp. Teor. Fiz. 58, 435 (1993) [JETP Lett. 58, 445 (1993)], Physica C 253, 373 (1995).

[8] S. N. Artemenko and A. G. Kobelkov, Phys. Rev. B 55, 9094 (1997).

[9] L. D. Landau and E. M. Lifshits, Statistical Physics (Nauka, Moscow, 1976).

[10] A. G. Aronov, V. L. Gurevich, Fiz. Tverd. Tela 16, 2656 (1974).

[11] T. Mishonov, Phys. Rev. B, 44, 12033 (1991); 50, 4004 (1994); M. Tachiki, S. Koyama, and M. Takahashi, Phys. Rev. B, 50, 7065 (1994).

[12] Ophelia K.C. Tsui, N.P. Ong, Y. Matsuda, and J.B. Peterson, Phys. Rev. Lett. 73, 724 (1994), Ophelia K.C. Tsui, N.P. Ong, and J.B. Peterson, Phys. Rev. Lett. 76, 819 (1996); Y. Matsuda, M.B. Gaifullin K. Kumagai, K. Kadowaki and T. Mochiku, Phys. Rev. Lett. 75, 4512 (1995).

[13] P.L. Carlson and A.M. Goldman, Phys. Rev. Lett. 34, 11 (1975).

[14] S.N. Artemenko, A.F. Volkov, JETP 69, 1764 (1975).

[15] A. Schmid and G. Schön, Phys. Rev. Lett. 34, 941 (1975).

[16] Yu.M. Ivanchenko, L.A. Zil'berman, JETP 55, 2395 (1968); V. Ambegaokar, B.I. Halperin, Phys. Rev. Lett. 
22, 1364 (1969).

[17] V.N. Zverev, D.V. Shovkun and I.G. Naumenko, JETP Letters. 68, 309 (1998). 